\title{
Use of linear feedback to control relaxation oscillations in a metal-passivation model
}

\author{
Alan J Markworth $\dagger$ and Anthony M Bloch $\ddagger$ \\ $\dagger$ Department of Materials Science and Engineering, The Ohio State University, Columbus, OH \\ 43210-1179, USA \\ $\ddagger$ Department of Mathematics, The University of Michigan, Ann Arbor, MI 48109-1003, USA
}

Received 7 July 1996, accepted for publication 26 August 1996

\begin{abstract}
A method of linear feedback is shown to be applicable to the control or suppression of spontaneously occurring relaxation oscillations in a two-dimensional model for the passivation of a metal surface exposed to an aqueous medium. For the operating conditions selected, these oscillations are characterized by a large limit cycle within the state space of the electrochemical system. A physically plausible control is used, involving feedback of a linear combination of two measurable quantities to a controllable parameter. The control acts to stabilize an otherwise unstable fixed point without changing its state space coordinates. Addition of the control to the system's dynamics also results in the appearance of two new fixed points, one of which is stable, the other unstable. The control is found to be extremely robust; when applied, even when the system is far from a fixed point, stabilization onto one or the other of the two stable fixed points occurs quickly. Which of the two it approaches depends upon the state space coordinates of the system on the limit cycle at the time the control is initiated. Stabilization onto the fixed point of the free system, from any point on the limit cycle, can be achieved using a combination of free and controlled dynamics. Release of the control causes the system to return to its oscillatory dynamics. Other linear-feedback-based approaches to control also are briefly considered, such as feeding back the time derivative of a measurable quantity to a controllable parameter.
\end{abstract}

\section{Introduction}

Electrochemical systems have long been known to exhibit spontaneous oscillatory behaviour under certain operating conditions. Depending upon the nature of a given system and its operating conditions, its oscillations may be periodic, quasiperiodic, or chaotic. Interest has recently arisen in strategies for exerting control over this oscillatory behaviour; for example, to make a chaotic electrochemical system oscillate periodically [1-3] or even exhibit steady-state behaviour [4], or to bring a periodically oscillating system to a steady state [4]. Success has been achieved in controlling oscillatory behaviour in numerical models for electrochemical systems $[1,3,4]$ and in actual electrochemical cells $[2,4]$.

In this work, we apply well known principles of linear control theory, in conjunction with the free nonlinear dynamics, to control the dynamical behaviour of a nonlinear model for the passivation of a metal surface exposed to an aqueous medium. Operating conditions selected for the model are such that its free (uncontrolled) dynamics consist of large-amplitude relaxation oscillations. It is shown that these oscillations can indeed be eliminated using a very simple feedback strategy. However, the particular control selected here for detailed study does add some unusual behaviour to the steady-state characteristics of the system, in the form of two additional fixed points. As in Bloch and Marsden [5], however, a 
combination of free dynamics and linear control can be used to stabilize the system onto the fixed point of the free system over the entire region enclosed by and including the stable manifold of the limit cycle. It is demonstrated that this control scheme has a surprising degree of robustness in that the application of the linear control to the full nonlinear system results in a large domain of attraction for the stabilized fixed point. Furthermore, while outside the domain of attraction, the nonlinear free dynamics is used to drive the system to the domain of attraction. Thus, while the initial analysis used to derive the control is based on the linear theory, the final control is a highly effective nonlinear one.

Other possible control strategies are also considered, albeit briefly. Emphasis here is placed on a method of 'derivative control', in which the derivative, with respect to time, of a measurable quantity is fed back to a controllable parameter.

Finally, it should be emphasized that the strategy used here to control an oscillating electrochemical system is, in principle, quite general and is also applicable to the control (or at least the attempt at control) of other types of nonlinear systems.

\section{The passivation model}

The passivation model that we use in this work was originally proposed by Talbot and Oriani [6], and was subsequently the subject of detailed analysis by other investigators [7,8]. Although the model is only two-dimensional (i.e. it is characterized by two independent state variables), it has been shown to exhibit extremely complex dynamics due to its highly nonlinear character. The precise nature of its dynamics depends upon the magnitude selected for the various rate constants. However, having only two dimensions, the model cannot exhibit chaotic oscillations, which require a minimum of three independent state variables.

The model is summarized in the appendix, and details regarding its complex dynamics have been described elsewhere [6-8]. Its two independent state variables are the fractional coverage, $\theta$, on the metal surface, of a passivating metal-hydroxide film, and the concentration, $Y$, of metal dissolved in the aqueous solution, expressed here in dimensionless form. These variables satisfy the following set of coupled, autonomous differential equations:

$$
\begin{aligned}
& \dot{\theta}=Y(1-\theta)-\theta \mathrm{e}^{-\beta \theta} \\
& \dot{Y}=p(1-\theta)-q Y
\end{aligned}
$$

where $p, q$, and $\beta$ are positive, dimensionless parameters, and the dot denotes differentiation with respect to a dimensionless time, $\tau$.

In this work, we consider the following set of values for the three variable parameters: $\{p, q, \beta\}=\{0.0002,0.001,5\}$. This choice is made on the basis of other work [7], in which it was shown that, for this model and for this particular set of parameter values, the stable dynamics consist of spontaneous, large-amplitude oscillations, characterized in state space by a large limit cycle. It is this highly nonlinear behaviour that we seek to control. Inside the limit cycle is a fixed point (an unstable node), having coordinates $\left\{\theta_{0}, Y_{0}\right\}=\{0.636924,0.072615\}$. The limit cycle and the fixed point are shown in figure 1, and the large-amplitude, oscillatory behaviour of $\theta$ is illustrated in figure 2. The numerical technique used to integrate equations (1) and (2) was a fourth-order Runge-Kutta method with fixed step size; the software package Mathcad PLUS 5.0 was used.

The dynamics, as illustrated in figures 1 and 2, constitute a type of 'relaxation oscillation', the general feature of which is that the system in question oscillates between two different states, switching abruptly from one to the other. The fact that such behaviour characterizes the present case is demonstrated in figure 3 , in which $\dot{\theta}$ is plotted as a function 


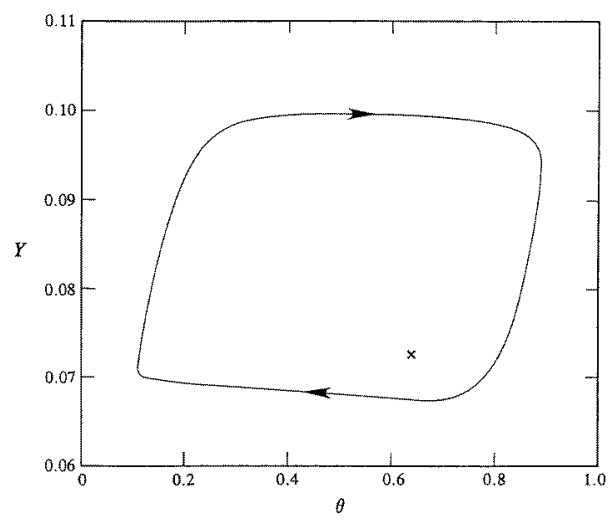

Figure 1. Illustration of the limit cycle exhibited by the Talbot-Oriani model for $\{p, q, \beta\}=$ $\{0.0002,0.001,5\}$. The arrows indicate the direction of 'flow' along the attractor and the $x$ denotes the location of the unstable node.

of $\tau$. It can be seen that the system spends most of the time, each cycle, situated near the $\dot{\theta}=0$ nullcline, a nullcline being a curve in state space along which the rate of change of one of the state variables is zero. The system jumps abruptly from a region near the nullcline for which $\dot{\theta}<0$ to one for which $\dot{\theta}>0$, then abruptly returning to $\dot{\theta}<0$, and so on.

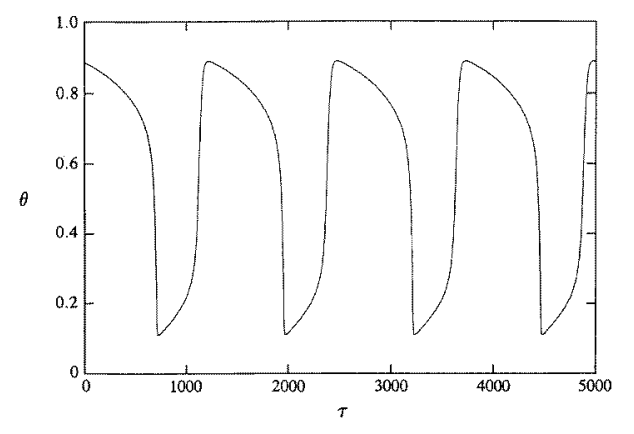

Figure 2. Relaxation oscillations, shown in a plot of $\theta$ against $\tau$, for the parameter values listed in figure 1.

\section{Demonstration of controllability}

In this section, we assess the applicability of linear control as a means with which to stabilize the fixed point, which, as we have noted, is an unstable node under uncontrolled conditions. Interestingly, the conclusion as to whether the system can be controlled by this method can be drawn without having to specify the precise nature of the control itself.

We begin by summarizing the classic linear control theory (see, for example, Bloch and Marsden [5] and references therein). Consider a linearized dynamical system expressed by

$$
\dot{\boldsymbol{x}}=\mathbf{A} \boldsymbol{x}+\mathrm{B} u
$$




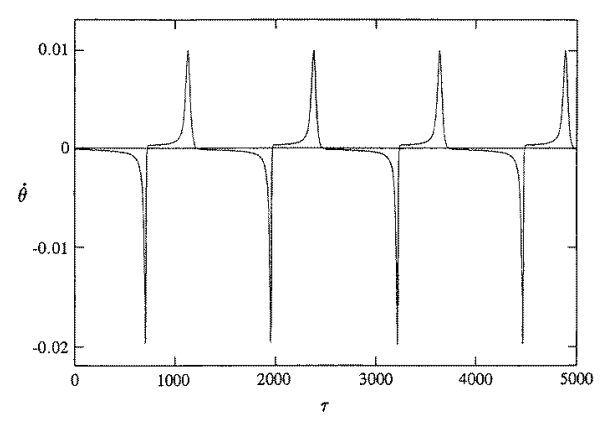

Figure 3. Variation of $\dot{\theta}$ with $\tau$ for the parameter values listed in figure 1 .

the dot denoting differentiation with respect to time. Here, $\boldsymbol{x}$ and $\boldsymbol{u}$ are vectors, the components of which are state variables and control parameters, respectively, with $\boldsymbol{x} \in \mathbb{R}^{n}$ and $\boldsymbol{u} \in \mathbb{R}^{m}$, and $\mathbf{A}$ and $\mathbf{B}$ are $n \times n$ and $n \times m$ matrices, respectively. This linearized system is said to be 'completely controllable' if and only if the $n \times n m$ matrix, C, called the controllability matrix, has rank $n$, where

$$
\mathbf{C}=\left[\mathbf{B}, \mathbf{A B}, \mathbf{A}^{2} \mathbf{B}, \ldots, \mathbf{A}^{n-1} \mathbf{B}\right] .
$$

We now apply this formalism to the corrosion model summarized in equations (1) and (2). First, we think in terms of applying a physically realistic control to the model. Toward this end, we note (see the appendix) that the parameter $p$ is associated with an electron-transfer reaction, and would generally change, with a change $\delta V$ of anodic potential, according to the relation

$$
p=p_{0} \exp (\alpha \delta V)
$$

where $p_{0}$ is the value of $p$ at $\delta V=0$ and $\alpha$ is a parameter which is temperature dependent and has units of inverse potential. Since the anodic potential is experimentally measurable, we take it to be the quantity to which the control is applied, so that the control effectively causes a change in the magnitude of $p$.

Next, we linearize the model about the fixed point by examining small perturbations therefrom, i.e.

$$
\theta=\theta_{0}+\eta(\tau) \quad Y=Y_{0}+\xi(\tau)
$$

The quantities $\eta$ and $\xi$ represent the first-order terms in Taylor-series expansions of $\theta$ and $Y$ about the fixed point. Taking $p_{0}$ to be the value of $p$ at the fixed point, we assume that its value changes, in the linear approximation, to

$$
p=p_{0}+u(\tau)
$$

due to some control, as yet unspecified, that is designed to restore the system to the fixed point, but which vanishes at the fixed point itself. The latter requirement ensures that the state space coordinates of the fixed point are not altered by the presence of the control. A necessary condition for such restoration is that the fixed point be stabilized. Combining equations (6) and (7) with (1) and (2), and retaining only terms of first order in $\eta$, $\xi$, and $u$, we obtain an expression that can be cast in the format of equation (3), with

$$
\boldsymbol{x}=\left(\begin{array}{l}
\eta \\
\xi
\end{array}\right) \quad \mathbf{A}=\left(\begin{array}{cc}
-Y_{0}+\left(\beta \theta_{0}-1\right) \mathrm{e}^{-\beta \theta_{0}} & 1-\theta_{0} \\
-p_{0} & -q
\end{array}\right)
$$




$$
\mathbf{B}=\left(\begin{array}{c}
0 \\
1-\theta_{0}
\end{array}\right) \quad \boldsymbol{u}=u
$$

noting that the vector $\boldsymbol{u}$ is simply a scalar in our case.

The $2 \times 2$ controllability matrix, $\mathbf{C}$, can be readily obtained from equations (4) and (8), and one can show that, for the values used here for $p_{0}, q$, and $\beta$, its determinant is non-zero. Therefore, since $\mathbf{C}$ does have rank 2 , the system is indeed controllable about the fixed point.

\section{Application of linear feedback}

The problem now is to select a physically plausible control, $u$, that will stabilize the fixed point. The linearized control that we seek will have the general form

$$
u=\alpha_{1} \eta+\alpha_{2} \xi
$$

where $\alpha_{1}$ and $\alpha_{2}$ are parameters determined by the physical quantities actually fed back to the anodic potential. The controlled, linearized system thus can be expressed as

$$
\dot{\boldsymbol{x}}=\mathbf{J} \boldsymbol{x}
$$

where $\mathbf{J}$ is the $2 \times 2$ Jacobian matrix, evaluated at the fixed point, the elements of which are determined from equations (3), (8), and (9). In order for the fixed point to be stabilized, the real parts of both eigenvalues of $\mathbf{J}$ must be negative. For a two-dimensional system such as this, this condition is satisfied if $\operatorname{tr}(\mathbf{J})<0$ and $\operatorname{det}(\mathbf{J})>0$. For $u=0$, one can show that, for the particular set of parameter values we are using, $\operatorname{tr}(\mathbf{J})>0$ and $\operatorname{det}(\mathbf{J})>0$, which illustrates the instability of the uncontrolled fixed point.

\subsection{Anodic-current feedback}

As a first attempt to achieve control, we feed back the change of anodic current, i.e.

$$
u=\gamma_{1} \delta j
$$

where $\delta j=j-j_{0}$, with $j$ being the current at the instantaneous state space configuration in which the system exists, $j_{0}$ being the current at the fixed point, and $\gamma_{1}$ is a variable control parameter. For this model, the current arises from the electron-transfer reaction associated with parameter $p$ (see equations (A.1) and (A.8) of the appendix), and is therefore proportional to the quantity $p(1-\theta)$. Taking the proportionality constant to be unity, and then noting that $j_{0}=p_{0}\left(1-\theta_{0}\right)$, one can show that, to first order in terms of $\eta$ and $u$,

$$
\delta j=\left(1-\theta_{0}\right) u-p_{0} \eta
$$

Combining equations (11) and (12), and solving for $u$,

$$
u=\frac{-\gamma_{1} p_{0} \eta}{1-\gamma_{1}\left(1-\theta_{0}\right)} .
$$

Using this linear (but complex) control in equation (8), we find that $\operatorname{tr}(\mathbf{J})$ remains unaltered, and thus is positive, regardless of the value of $\gamma_{1}$. Therefore, this control cannot, by itself, stabilize the fixed point. 


\subsection{Solution-concentration feedback}

As a second attempt to achieve control, we choose

$$
u=\gamma_{2} \delta Y
$$

where $\delta Y=Y-Y_{0}$, with $Y$ being the solution concentration at the instantaneous state space position, $Y_{0}$ being its value at the fixed point, and $\gamma_{2}$ is another variable control parameter. In the linear approximation, $\delta Y=\xi$, so that $u=\gamma_{2} \xi$. With this control, one can show that there is no value of $\gamma_{2}$ for which the conditions $\operatorname{tr}(\mathbf{J})<0$ and $\operatorname{det}(\mathbf{J})>0$ are simultaneously satisfied. Consequently, as was the case with the $\delta j$ feedback, this control cannot, by itself, stabilize the fixed point.

\subsection{Linear-combination feedback}

Although neither $\delta j$ nor $\delta Y$ was a suitable feedback variable to the parameter $p$, it so happens that appropriate linear combinations of these variables $d o$ stabilize the fixed point. To demonstrate this fact, we set

$$
u=\gamma_{1} \delta j+\gamma_{2} \delta Y .
$$

Substituting our linearized expressions for $\delta j$ and $\delta Y$ (equations (12) and (6), respectively) into equation (15), and solving for $u$, we obtain

$$
u=\frac{-\gamma_{1} p_{0} \eta+\gamma_{2} \xi}{1-\gamma_{1}\left(1-\theta_{0}\right)} \text {. }
$$

For the parameter values selected here, one can show that the fixed point is stabilized if the following expression is satisfied:

$$
-\frac{8.472 \times 10^{-3}+10^{-3} \gamma_{1}}{1-0.3631 \gamma_{1}}<\frac{\gamma_{2}}{1-0.3631 \gamma_{1}}<-0.04631 .
$$

The inequality in expression (17) is derived simply by requiring that the relationships $\operatorname{tr}(\mathbf{J})<0$ and $\operatorname{det}(\mathbf{J})>0$ are simultaneously satisfied. For present purposes, we choose just one set of values for which stabilization is achieved: $\left\{\gamma_{1}, \gamma_{2}\right\}=\{2.5,-0.01\}$, for which the fixed point can be shown to be a stable node. This choice was made largely on the basis of a trial-and-error search for parameter values that would stabilize the fixed point. One can verify, however, that expression (17) is indeed satisfied when these values are substituted therein. In fact, one can show, by way of example, that for $\gamma_{1}=2.5$, expression (17) is satisfied as long as $\gamma_{2}$ lies within the range $-0.01098<\gamma_{2}<-0.004275$. Also, for this particular set of $\left\{\gamma_{1}, \gamma_{2}\right\}$ values, the coefficients of both $\eta$ and $\xi$ in equation (16) are negative, so that the feedback itself is negative. Of course, any set of $\left\{\gamma_{1}, \gamma_{2}\right\}$ values for which equation (17) is satisfied will serve to stabilize the fixed point.

In section 5, we investigate the consequences of applying this control to the system, which, uncontrolled, would undergo the relaxation oscillations illustrated in figures 1-3, but first we briefly consider some other possibilities for oscillation control based on linear feedback.

\subsection{Other feedback possibilities}

The feedback strategies summarized above all involved taking some measurable quantity, or a linear combination of measurable quantities, and feeding it back to a controllable parameter. The controllable parameter we selected was $p$, because of its dependence upon the adjustable electrode potential (see equation (5)). One might also regard $q$ as 
a controllable parameter, assuming there is indeed some experimental means by which it can be adjusted. In that case, one would have to re-do the test for controllability, this time taking $q=q_{0}+u(\tau)$, where $q_{0}$ is the value of $q$ at the fixed point. Another strategy, one again that is experimentally realizable, involves feeding back the time derivative of a measurable quantity to a controllable parameter, an approach recently used by Parmananda et al [4]. We shall consider one example of this so-called 'derivative control', namely, feeding back $\dot{Y}$ to the parameter $p$.

We now set

$$
u=\gamma_{3} \dot{Y}
$$

where $\gamma_{3}$ is another control parameter. Substituting the linearized forms for $\theta, Y$, and $p$, given in equations (6) and (7), into equation (2), and combining the result with equation (18), we solve for $u$ to obtain,

$$
u=\frac{-\gamma_{3}\left(p_{0} \eta+q \xi\right)}{1-\gamma_{3}\left(1-\theta_{0}\right)} \text {. }
$$

Equation (19) clearly has some similarity to (16), although there is now only one adjustable control parameter. Nevertheless, we do find, again by trial-and-error, that there are indeed values for $\gamma_{3}$ for which the fixed point is stabilized. For example, for $\gamma_{3}=2.74$ the fixed point is a stable focus, whereas for $\gamma_{3}=2.75$ it is a stable node.

One interesting feature of derivative control is that it can add a new dimension to the system, depending upon the quantity selected for feedback. Such was found to be the case in the work of Parmananda et al [4]. For example, were we to feed back to $p$ the time derivative of the current $j$, then the parameter $p$ would actually have to be treated as a third variable, and we would have an expression for $\dot{p}$ that would be treated as a third rate equation, coupled to equations (1) and (2).

\section{Results}

\subsection{Additional fixed points}

Application of the control given by equation (16) does stabilize the fixed point without changing its coordinates in state space, since the control itself vanishes at the fixed point. However, an interesting feature of this control is that, for the set of $\left\{\gamma_{1}, \gamma_{2}\right\}$ values used here, its addition to the dynamics of the system causes the appearance of two new fixed points within the limit cycle. One of these is a stable focus, located at $\left\{\theta_{1}, Y_{1}\right\}=\{0.222419,0.09407\}$, which is quite close to points on the limit cycle. The other is an unstable node, located at $\left\{\theta_{2}, Y_{2}\right\}=\{0.61386,0.073849\}$, which is close to $\left\{\theta_{0}, Y_{0}\right\}$. The system would be stable, situated at $\left\{\theta_{1}, Y_{1}\right\}$, but the control does not vanish there as it does at $\left\{\theta_{0}, Y_{0}\right\}$, which therefore makes it undesirable from an energetic standpoint.

The appearance of the new fixed points is explained by examination of the nullclines. For a two-dimensional model, an intersection of the two nullclines is a fixed point. Nullclines for this model are shown in figure 4. That for which $\dot{\theta}=0$ does not depend on $p$, and so is independent of the applied control. However, that for which $\dot{Y}=0$ is control dependent, and is shown in figure 4 for both the uncontrolled and the controlled cases. Clearly, only one fixed point exists if the system is uncontrolled, whereas there are three for the controlled case, due to the control-induced change in the $\dot{Y}=0$ nullcline.

One might question whether the appearance of the additional fixed points may be an artifact of the use of linear feedback in regions of state space that are far removed from the point $\left(\theta_{0}, Y_{0}\right)$, about which the system had been linearized. To settle this issue, we consider 
the more physically realistic (but far more complex) case of feeding back to the anodic potential using the nonlinear exponential relation given in equation (5), setting

$$
\alpha \delta V=\frac{1}{p_{0}}\left(\gamma_{1} \delta j+\gamma_{2} \delta Y\right)
$$

from which we obtain

$$
\alpha \delta V=\frac{1}{p_{0}}\left\{\gamma_{1}\left[p(1-\theta)-p_{0}\left(1-\theta_{0}\right)\right]+\gamma_{2}\left(Y-Y_{0}\right)\right\} .
$$

The complexity associated with application of this form of exponential feedback arises from the fact that the parameter $p$ is actually being fed back to itself. One can use equations (5) and (21) to show that the first-order, feedback-induced correction to $p_{0}$ is consistent with equation (16). In addition, this more realistic relation for $p$ can be used to calculate the correspondingly new nullcline for $\dot{Y}=0$ (using equation (2)), i.e.

$$
\theta=1-\left(\frac{q Y}{p_{0}}\right) \exp \left[-\frac{1}{p_{0}}\left(\gamma_{1} q+\gamma_{2}\right)\left(Y-Y_{0}\right)\right] .
$$

Nullclines for both the linear feedback and the exponential feedback are plotted in figure 5. The two $\dot{Y}=0$ nullclines follow the same general trend, but, as expected, differ more significantly from one another at state space positions far removed from $\left\{\theta_{0}, Y_{0}\right\}$. The important point, however, is that even for the case of exponential feedback, the appearance of two new fixed points still does occur, and thus is not an artifact of the use of linear feedback.

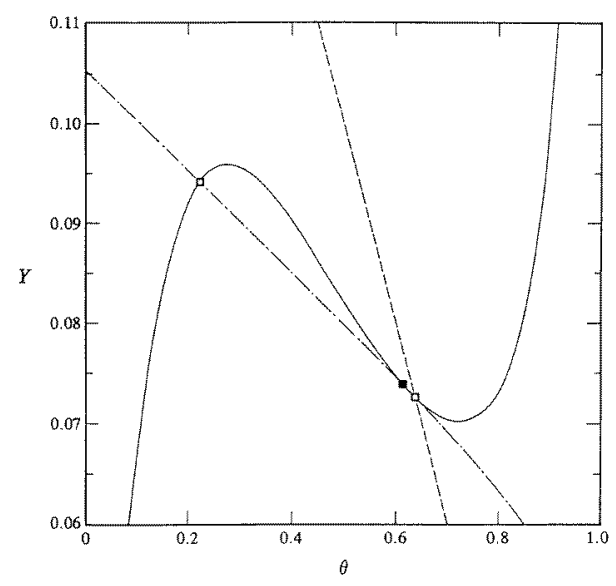

Figure 4. Effects of control on nullclines and fixed points. The dashed curve is for $\dot{Y}=0$ with no applied control; the dot-dashed curve is for $\dot{Y}=0$ with linear control; the full curve is for $\dot{\theta}=0$. The two stable fixed points are shown as open squares, the unstable fixed point as a full square.

One could, of course, seek to eliminate the control-induced fixed points, for example, by varying $\gamma_{1}$ and $\gamma_{2}$ or by attempting to use other feedback variables. That possibility is not investigated here. In fact, the dynamical behaviour associated with the particular control system we have selected is quite interesting, as demonstrated in the following section.

It should be noted that equations (5) and (21) do not uniquely determine the value of $p$, for given $\theta$ and $Y$. In general, there are two values of $p$ that satisfy these expressions, but it is the smaller of the two that converges to $p_{0}$ as $\theta$ and $Y$ approach their fixed-point values. 


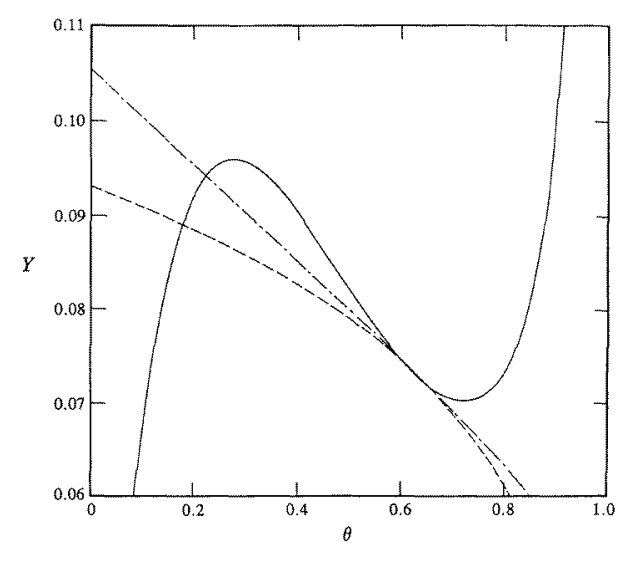

Figure 5. Comparison of $\dot{Y}=0$ nullclines for exponential feedback (dashed curve) and linear feedback (dot-dashed curve). The full curve is the nullcline $\dot{\theta}=0$.

\subsection{Control or suppression of oscillations}

The question remains as to whether the attractor for the free system (that is, the large limit cycle) lies within the basin of attraction of one or the other, or possibly both, of the two control-induced stable fixed points. To answer this question, the control (using the simpler, linear feedback) was initiated at various different locations on the limit cycle, and the subsequent dynamics calculated. The results are illustrated in figure 6. Clearly, the control is quite effective in eliminating the limit cycle; however, it can be seen that which of the two stable fixed points the system falls to depends on the location of the system on the attractor when the control is initiated. The basin of attraction for the new stable fixed point is seen to be quite extensive, actually reaching to regions of state space that are relatively close to the original, but now stabilized, fixed point. As in Bloch and Marsden [5], this suggests the following strategy for a stabilizing controller. Let $u=0$, so that the free dynamics drives the system until it enters what would be, under the influence of the control, the basin of attraction for the fixed point of the free system. Then initiate the control.

It is interesting that, even though the system is fundamentally nonlinear, satisfactory control can be achieved by using the linear theory of control and the nonlinear free dynamics, as was the case in Bloch and Marsden [5]. However, it should be noted that, while the linear theory was used to design the control, the controlled system is taken to initially lie on the limit cycle, and is therefore highly nonlinear. The applied control consisted of combining equations (7) and (16), and substituting the result into (2), setting $\eta=\theta-\theta_{0}$ and $\xi=Y-Y_{0}$. In this initially nonlinear regime, $\eta$ and $\xi$ are not just of first order, but include higher-order contributions as well. The fact that the control is effective, even under these highly nonlinear conditions, demonstrates that it is extremely robust.

In figure 7, the time dependence of $\theta$ is illustrated, initially with no control. Then, the control is applied and the system quickly falls to the now-stabilized fixed point (not, in this case, to the control-induced fixed point). When the control is then turned off, the system remains near the fixed point for a period of time, but then returns to its largeamplitude oscillations. The state space portrait for this sequence of events is presented in figure 8 . Finally, the corresponding variation of $u / p_{0}$ is plotted in figure 9 . It can be seen that, for a brief period of time near the initiation of control, during which the linearization 


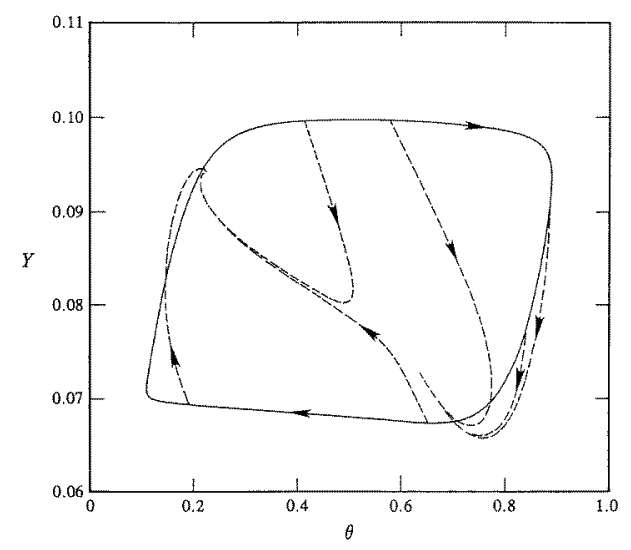

Figure 6. A phase portrait illustrating the effects of initiating control at different points on the limit cycle. The full curve represents the uncontrolled limit cycle, whereas the dashed curves represent behaviour after the initiation of control.

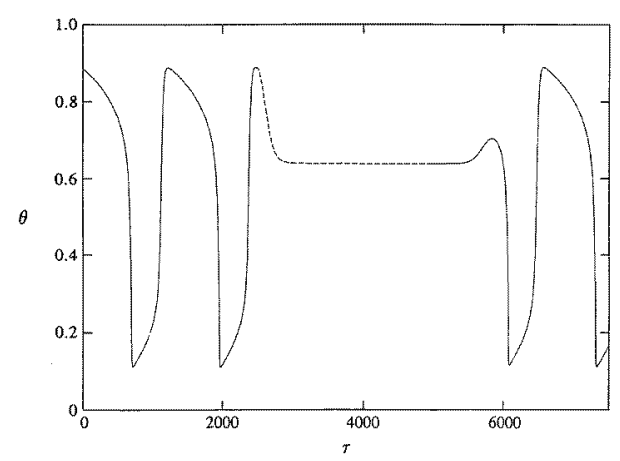

Figure 7. Variation of $\theta$ with $\tau$. Initially, there is no control (full curve). Then the control is applied (dashed curve). Finally, the control is removed (full curve).

approximation is certainly not valid, this ratio deviates significantly from zero, actually reaching a non-physical value of about -17 immediately upon application of the control. However, the system soon recovers and, for most of the time over which the control is applied, the ratio has a value close to zero.

\section{Conclusion}

Control of relaxation oscillations in a two-dimensional model of metal passivation has been shown to be possible using linear feedback of a combination of two measurable quantities to a controllable parameter. It was also found that the control itself, while stabilizing a preexisting fixed point and leaving its state space coordinates unchanged, causes the formation of two new fixed points, one of which is stable, the other unstable. Upon application of the control, the system leaves the limit cycle and falls into one or the other of the two stable fixed points. Which of the two it approaches depends upon its instantaneous state space coordinates at the time the control is first applied. 


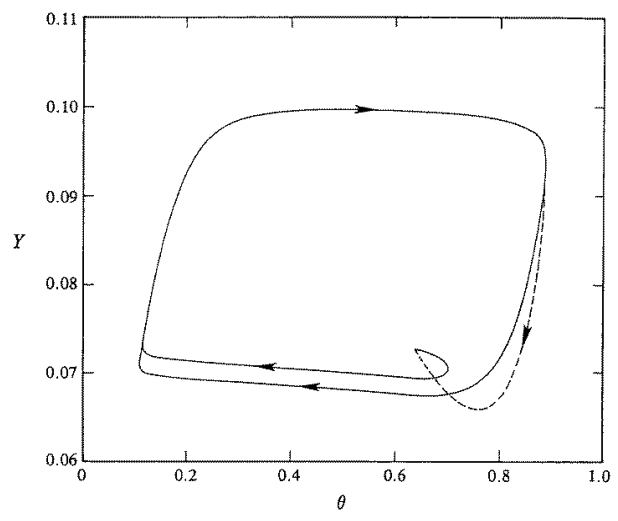

Figure 8. State space portrait for the sequence of events depicted in figure 7. Again, the full curves represent no control; the dashed curve is for the controlled system.

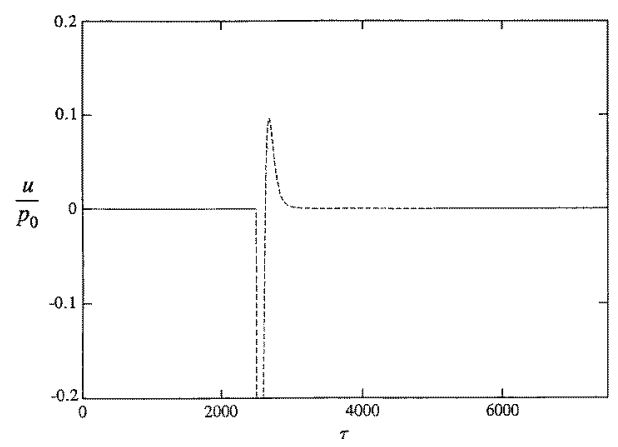

Figure 9. Plot of $u / p_{0}$ against $\tau$ for the sequence of events shown in figure 7 .

As far as the control-induced stable fixed point is concerned, it would not be energetically favourable for stabilization to be experimentally carried out on such a point, since the magnitude of the control does not vanish as it does at the original fixed point. Stabilization onto the fixed point for the free system can be achieved, as described above, by a combination of free and controlled dynamics. The control can sequentially be 'turned on' and 'turned off' until the desired fixed point is reached. In this regard, the overall control strategy is actually nonlinear.

Use of the $\dot{Y}$ derivative control was also found to be successful in stabilizing the fixed point, and likewise in suppressing the oscillations, although the latter calculations were not presented here.

Although the control methods described in this work were applied only to a mathematical model, the general strategies should be applicable experimentally, since they were used here in a physically realistic manner. One disparity would be that the control fed back experimentally would not be the linear correction, $u$, but rather would involve the full exponential dependence of what in this case is the anodic current upon the applied potential. However, at least for the example we have seen, the difference between the linear and exponential cases is not significant except at the earliest times following application of the control. 
Of course, still other control strategies also could be developed and used to stabilize a fixed point. The final choice, particularly in an experimental situation, would be based on relative ease of application of a given strategy and its robustness.

\section{Acknowledgments}

AJM was supported by the Electric Power Research Institute (EPRI), with particular thanks being expressed to EPRI's Dr John Stringer. The work was begun at Battelle Memorial Institute in Columbus, under EPRI contract RP2426-25, and completed at The Ohio State University under EPRI contract WO9000-35. AMB was supported under AFOSR grants F49620-93-1-0037 and F49620-96-1-0100, NSF PYI grant DMS-91-57556, and a Guggenheim Fellowship.

\section{Appendix. The metal-passivation model}

The Talbot-Oriani model [6], for the passivation of a metal surface exposed to an aqueous medium, is characterized by the following set of reactions:

$$
\begin{aligned}
& \mathrm{M} \underset{k_{1}}{\rightarrow} \mathrm{M}^{+}+\mathrm{e}^{-} \\
& \mathrm{M}^{+}+\mathrm{H}_{2} \mathrm{O} \underset{k_{-2}}{\stackrel{k_{2}}{\rightleftarrows}} \mathrm{MOH}_{\mathrm{aq}}+\mathrm{H}^{+} \\
& n \mathrm{M}^{+}+\mathrm{A}^{n-} \underset{k_{r}}{\rightarrow} \mathrm{M}_{n} \mathrm{~A} \\
& \mathrm{MOH}_{\mathrm{aq}} \underset{k_{-3}}{\stackrel{k_{3}}{\rightleftarrows}} \mathrm{MOH}_{\mathrm{ad}}
\end{aligned}
$$

where $\mathrm{M}$ is the metal species, $\mathrm{A}^{n-}$ is some anion in the aqueous medium other than $\mathrm{OH}^{-}$, and the $k$ 's are characteristic rate constants. The adsorbed $\mathrm{MOH}_{\mathrm{ad}}$ film passivates the metal surface, that is, prevents further dissolution of the metal.

This model has two independent state variables: the fractional coverage, $\theta$, of the passivating metal-hydroxide film and $\left[\mathrm{M}^{+}\right]$, the concentration of metal (taken to be monovalent) in solution. In the various studies involving this model [6-8], the parameter $k_{-3}$ was taken to vary exponentially with $\theta$, such that

$$
k_{-3}=k_{-3}^{\prime} \exp (-\beta \theta)
$$

where $\beta$ is a positive, dimensionless constant.

Taking $n=1$, and assuming the hydrolysis reaction is always in equilibrium, the rate equations can be expressed in dimensionless form as [6-8]

$$
\begin{aligned}
& \dot{\theta}=Y(1-\theta)-\theta \mathrm{e}^{-\beta \theta} \\
& \dot{Y}=p(1-\theta)-q Y
\end{aligned}
$$

where all the variables and parameters are dimensionless, with

$$
\begin{array}{lcc}
p \equiv K k_{1} / k_{-3}^{\prime} & q \equiv k_{r}^{\prime} / k_{-3}^{\prime} & Y \equiv K\left[\mathrm{M}^{+}\right] \\
K \equiv k_{3}^{\prime} / k_{-3}^{\prime} & \tau \equiv k_{-3}^{\prime} t &
\end{array}
$$

with the dot denoting differentiation with respect to $\tau$, and with $k_{r}^{\prime}$ and $k_{3}^{\prime}$ being proportional to $k_{r}$ and $k_{3}$, respectively. 


\section{References}

[1] Rollins R W, Parmananda P and Sherard P 1993 Phys. Rev. E 47 R780

[2] Parmananda P, Sherard P, Rollins R W and Dewald H D 1993 Phys. Rev. E 47 R3003

[3] Rhode M A, Rollins R W and Vassiliadis C A 1994 Proc. 26th Southeastern Symp. on System Theory (Los Alamitos, CA: IEEE Computer Society) p 638

[4] Parmananda P, Rhode M A, Johnson G A, Rollins R W, Dewald H D and Markworth A J 1994 Phys. Rev. E 495007

[5] Bloch A M and Marsden J E 1989 Theor. Comput. Fluid Dynamics 1179

[6] Talbot J B and Oriani R A 1985 Electrochim. Acta 301277

[7] Markworth A J, McCoy J K, Rollins R W and Parmananda P 1992 Applied Chaos ed J H Kim and J Stringer (New York: Wiley) p 277

[8] Markworth A J, McCoy J K and Glasser M L 1992 Scr. Metall. Mater. 271801 